\title{
Cumulative fluid accumulation is associated with the development of acute kidney injury and non-recovery of renal function: a retrospective analysis
}

Jing Zhang ${ }^{1,2}$, Siobhan Crichton ${ }^{3}$, Alison Dixon ${ }^{4}$, Nina Seylanova ${ }^{1,5}$, Zhiyong Y. Peng ${ }^{2}$ and Marlies Ostermann ${ }^{1 *}$ (D)

\begin{abstract}
Background: Acute kidney injury (AKI) is common in patients in the intensive care unit (ICU) and may be present on admission or develop during ICU stay. Our objectives were (a) to identify factors independently associated with the development of new AKI during early stay in the ICU and (b) to determine the risk factors for non-recovery of AKI.
\end{abstract}

Methods: We retrospectively analysed prospectively collected data of patients admitted to a multi-disciplinary ICU in a single tertiary care centre in the UK between January 2014 and December 2016. We identified all patients without AKI or end-stage renal failure on admission to the ICU and compared the outcome and characteristics of patients who developed AKI according to KDIGO criteria after $24 \mathrm{~h}$ in the ICU with those who did not develop AKI in the first 7 days in the ICU. Multivariable logistic regression was applied to identify factors associated with the development of new AKI during the 24-72-h period after admission. Among the patients with new AKI, we identified those with full, partial or no renal recovery and assessed factors associated with non-recovery.

Results: Among 2525 patients without AKI on admission, the incidence of early ICU-acquired AKI was 33.2\% (AKI I 41.2\%, AKI II 35\%, AKI III 23.4\%). Body mass index, Sequential Organ Failure Assessment score on admission, chronic kidney disease (CKD) and cumulative fluid balance (FB) were independently associated with the new development of AKI. By day 7,69\% had fully recovered renal function, $8 \%$ had partial recovery and $23 \%$ had no renal recovery. Hospital mortality was significantly higher in those without renal recovery. Mechanical ventilation, diuretic use, AKI stage III, CKD, net FB on first day of AKI and cumulative FB $48 \mathrm{~h}$ later were independently associated with nonrecovery with cumulative fluid balance having a U-shape association.

Conclusions: Early development of AKI in the ICU is common and mortality is highest in patients who do not recover renal function. Extreme negative and positive FB were strong risk factors for AKI non-recovery.

Keywords: Acute kidney injury, Recovery, Cumulative fluid balance

\footnotetext{
* Correspondence: Marlies.Ostermann@gstt.nhs.uk

'Department of Critical Care, King's College London, Guy's and St Thomas'

NHS Foundation Trust, London SE1 7EH, UK

Full list of author information is available at the end of the article
}

(C) The Author(s). 2019 Open Access This article is distributed under the terms of the Creative Commons Attribution 4.0 International License (http://creativecommons.org/licenses/by/4.0/), which permits unrestricted use, distribution, and reproduction in any medium, provided you give appropriate credit to the original author(s) and the source, provide a link to the Creative Commons license, and indicate if changes were made. The Creative Commons Public Domain Dedication waiver (http://creativecommons.org/publicdomain/zero/1.0/) applies to the data made available in this article, unless otherwise stated. 


\section{Background}

Acute kidney injury (AKI) is common during critical illness, affecting $>50 \%$ of patients in the intensive care unit (ICU) [1]. It is a syndrome rather than a defined diagnosis, has many different aetiologies and can develop at different stages throughout critical illness [2]. There is increasing evidence that AKI is associated with shortand long-term complications and high healthcare costs [3]. Patients who leave hospital alive remain at risk of chronic health problems, including chronic kidney disease (CKD) and end-stage renal disease (ESRD) [4, 5]. The risk is particularly high in patients with more severe, recurrent or prolonged AKI and in those with reduced renal functional reserve.

Many patients already suffer from AKI when admitted to the ICU but a large proportion develop AKI later whilst receiving critical care [6]. Their risk of dying is significantly higher than that of patients with AKI on admission. There is a clear need for better strategies to prevent the development of AKI during critical illness and to reduce the risk of progression in those who develop AKI. Although some risk factors are not modifiable, for instance advanced age or pre-existing CKD, others are avoidable or potentially amenable to modification, for instance exposure to nephrotoxic drugs [7-9]. A better understanding of the risk of ICU-acquired AKI and the identification of potentially modifiable risk factors is essential to reduce the global burden of AKI.

The objectives of this study were (a) to identify factors associated with the development of new AKI during early stay in the ICU and (b) to ascertain risk factors for non-recovery of AKI.

\section{Methods}

\section{Setting}

Guy's and St Thomas' NHS Foundation Hospital is a tertiary care centre with a 54-bed consultant-led multidisciplinary ICU. The ICU has a fully computerized electronic patient record system where all data are recorded at the time of generation.

\section{Patients and study design}

We analysed the departmental database containing prospectively collected data of adult patients ( $\geq 18$ years) admitted to the ICU between January 2014 and December 2016 and identified all patients with AKI as defined by the urine and creatinine criteria of the Kidney Disease Improving Global Outcome (KDIGO) classification [9]. In cases where baseline renal function was not available, we retrospectively imputed the serum creatinine using the Modification of Diet in Renal Disease (MDRD) equation, as suggested by the KDIGO expert group [9].

In our ICU, serum creatinine is measured on admission to the ICU, routinely every morning at around 6 a.m. and in-between as directed by the clinical team. Urine output is monitored and recorded hourly. To identify factors associated with the new development of AKI, we only analysed patients who did not have AKI in the first 24-h time period after ICU admission [10]. Therefore, we excluded patients with (i) AKI on admission or within the first $24 \mathrm{~h}$ of admission to the ICU, (ii) known ESRD, (iii) a renal transplant, (iv) renal replacement therapy (RRT) prior to ICU admission and (v) admission to the ICU after a nephrectomy. Other exclusion criteria were previous admission to the ICU during the same hospitalization, ICU stay $<48 \mathrm{~h}$ and pregnancy. We compared patients who developed AKI between the 24-72-h time period after admission to the ICU with patients who did not develop AKI within 7 days after admission to the ICU.

Among patients with AKI acquired in the ICU, we determined the degree of renal recovery at day 7 after ICU admission and distinguished between (a) full renal recovery (i.e. return of serum creatinine to baseline and urine output $>0.5 \mathrm{ml} / \mathrm{kg}$ ), (b) partial renal recovery (i.e. improvement of AKI to lower stage but no return to baseline) and (c) no renal recovery. Patients who were receiving RRT on day 7 were classified as having "no renal recovery"; patients who had received RRT in the first 6 days in the ICU and were no longer on RRT on day 7 were classified as having "partial recovery" independent of serum creatinine concentration. We also collected serum creatinine results at hospital discharge and investigated a change from baseline.

\section{Data collection}

We extracted the following data: demographics, chronic co-morbidities, Sequential Organ Failure Assessment (SOFA) score on admission to the ICU and on the first day of AKI, daily haemodynamic variables and serum creatinine for 7 days after admission to the ICU and at hospital discharge. Daily cumulative fluid balance was calculated automatically in our electronic medical records and defined as the difference between the total fluid intake from all sources (intravenous fluids, blood products, enteral and parenteral nutrition and medications) and total output (urine, effluent, output from drains and gastrointestinal losses). We recorded daily cumulative fluid balance in millilitres/24 $\mathrm{h}$ and percentage body weight (BW \%). We extracted data on the type of organ support, use of diuretics and exposure to specific nephrotoxic medications (aminoglycosides, non-steroidal anti-inflammatory drugs, including paracetamol, chemotherapy, contrast, vancomycin and antiretroviral drugs).

In the cohort of patients who developed new AKI during the 24-72-h period in the ICU, we identified the median day of AKI development and used this day as the reference point for comparison with the non-AKI group. 
To identify risk factors for non-recovery of renal function, we distinguished between factors prior to the development of new AKI and factors post-onset of AKI. The main outcomes were the development of new AKI, renal function on day 7 or day of hospital discharge (whatever occurred first) and survival status at the ICU and hospital discharge.

\section{Statistical analysis}

Continuous variables were summarized as mean and standard deviation (SD) or median and interquartile range (IQR) and compared between those who did and did not develop AKI in the first $24-72 \mathrm{~h}$ in the ICU, and those who did and did not recover from AKI, using the independent-samples $t$ test or Mann-Whitney $U$ test, as appropriate. Categorical variables were summarized as frequencies and percentages and compared using the chi-squared test. In the first multivariable model, the relationships between odds of developing AKI and demographic and clinical characteristics significant in univariable analyses were explored using logistic regression. Variables with small sample sizes (e.g. epinephrine use) and variables that were highly colinear with other variables (e.g. CKD and baseline serum creatinine) were not included in the multivariable analyses. Fractional polynomials were used to model the non-linear relationship between fluid balance and risk of AKI. In patients with AKI, multivariable logistic regression models were also used to explore the relationship between odds of non-recovery and (a) variables known on the first day of AKI only and (b) variables representing conditions after the development of AKI only. Fractional polynomials were again used to explore the effect of cumulative fluid balance in model (1) and net fluid balance on day of AKI (2). Survival analysis was used to describe cumulative hospital survival. $p$ values $<0.05$ were considered

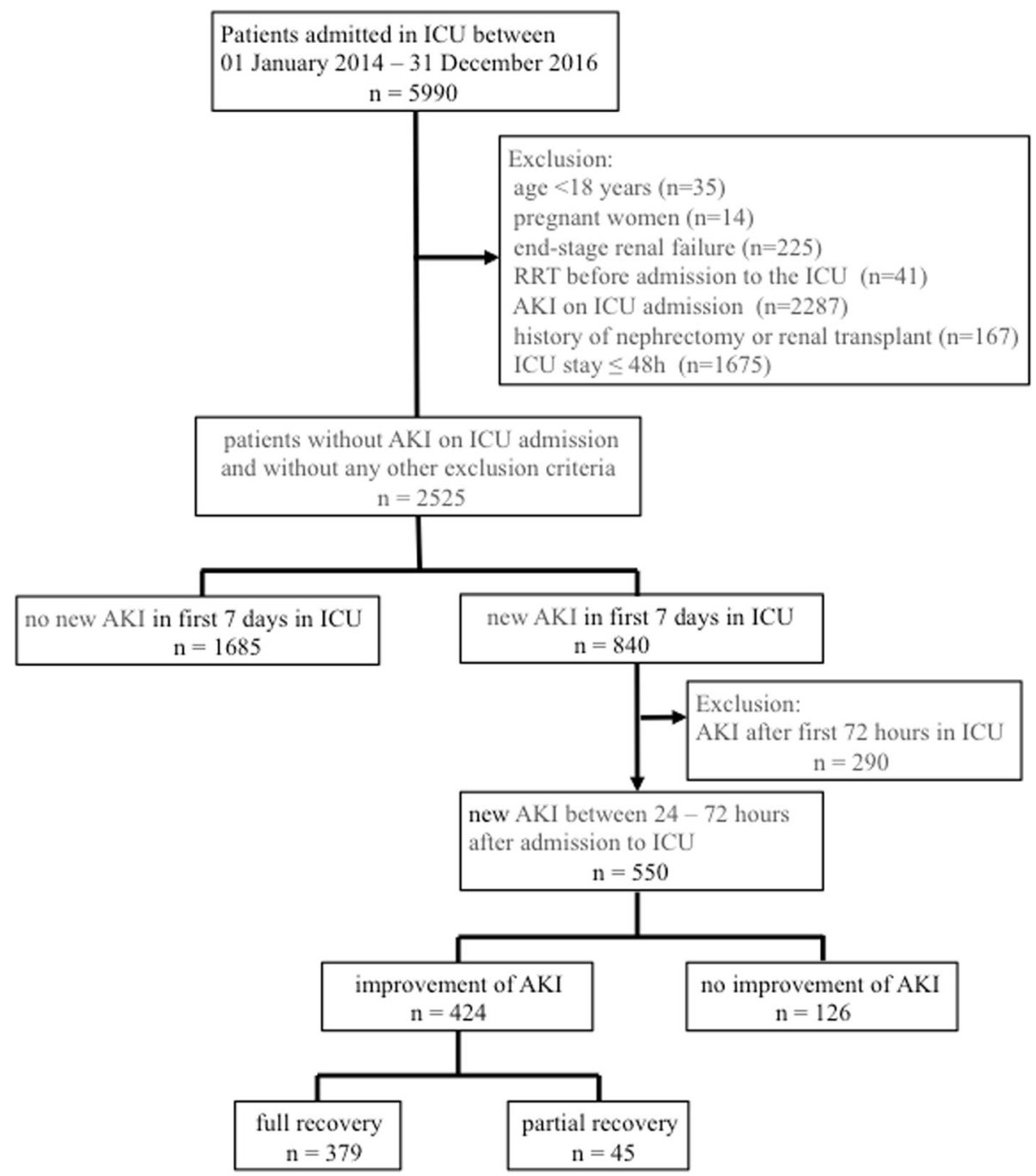

Fig. 1 Patient flow. AKI acute kidney injury, ICU intensive care unit, RRT renal replacement therapy 
statistically significant. Statistical analyses were performed using IBM SPSS Statistics 20.0 and STATA 15/ IC.

\section{Results}

Between January 2014 and December 2016, 5990 patients were admitted to the ICU; 2525 patients did not have AKI on admission and did not meet any exclusion criteria (Fig. 1). Among this cohort, 840 (33\%) patients developed new AKI at median day 3 (IQR 2-4) compared to 1685 (67\%) patients who did not develop AKI during the first 7 days in the ICU. The majority of patients with new AKI had AKI stage I (41\%) followed by AKI stage II (35\%) and AKI stage III (24\%). Patients who developed new AKI were older and characterized by a significantly higher SOFA score and higher CVP on admission to the ICU, a higher prevalence of pre-existing CKD and cardiovascular disease, greater need for advanced organ support, higher cumulative fluid balance and longer periods of inotrope and/or vasopressor support compared to patients without new AKI. (Table 1).

Among all patients with new AKI, 550 patients developed AKI during the 24- to 72-h period after admission to the ICU. Their ICU mortality was $19.8 \%$ and hospital mortality was $32.2 \%$. Among this cohort, 379 (69\%) had full recovery of renal function during the 7-day period after admission to the ICU, 45 (8\%) had partial renal recovery, and in 126 (23\%) patients, renal function did not improve (Fig. 1 and Additional file 1). Patients without renal recovery were characterized by a higher SOFA score and higher CVP prior to the development of AKI and in the following 24-48 h (Additional file 1 and Additional file 2). In addition, a higher proportion of AKI patients with non-recovery were treated with extracorporeal membrane oxygenation (ECMO), mechanical ventilation and/or noradrenaline following the development of AKI suggesting that they had more associated organ failure. The risk of non-recovery was higher in patients with AKI stage III (Additional file 2). Finally, among 177 patients with new AKI who died in the hospital, $71(40 \%)$ had no recovery of renal function compared to 56 of 373 patients (15\%) with new AKI who were discharged from the hospital alive.

\section{Multivariable analyses}

Multivariable logistic regression analysis showed that BMI, SOFA score on admission to the ICU, pre-existing CKD and cumulative FB on day of AKI or day 3 were independently associated with new AKI (Table 2). In a separate model including cumulative fluid balance in the first $24 \mathrm{~h}$, fluid balance was not associated with risk of developing AKI $(\mathrm{OR}=0.98,95 \% \mathrm{CI}, 0.96-1.01$, $p=0.159$ ).
In patients with new AKI, risk factors for nonrecovery within the first 7 days included factors before and after the onset of new AKI. Independent pre-AKI risk factors for non-recovery were CKD, need for mechanical ventilation, use of diuretics and net FB on the first day of AKI (Table 3 and Fig. 2a). Severity of AKI, mechanical ventilation and cumulative FB in the 48 - $h$ period after the day of AKI were independent post-AKI risk factors for non-recovery of renal function (Table 3 and Fig. 2b).

Both net FB and cumulative FB showed an independent U-shape association with renal recovery (Fig. 2a and b). In sensitivity analyses in which models were fitted separately to those who died in the hospital and those discharged alive, the U-shape relationship was present in both groups (Additional file 5: Figure $\mathrm{S} 1$ and Additional file 6: Figure S2). There were 37 patients with a cumulative $\mathrm{FB}$ of $-5 \mathrm{l}$ or more on the day of AKI or day 3 in the ICU (in patients without AKI); they were less likely to be male, more likely to have congestive heart failure, chronic lung disease, a respiratory diagnosis, to be on ECMO and to have received a diuretic than those with cumulative FB less than - 51 (Additional file 3). There were also 271 patients with a cumulative $\mathrm{FB}$ greater than +51 which was associated with lower MAP, higher baseline serum creatinine, known cardiovascular disease, gastrointestinal and sepsis diagnoses on admission and the use of norepinephrine.

\section{Outcomes}

Hospital mortality was significantly higher in patients who developed AKI in the ICU compared to those without AKI (35.4\% versus $15.4 \%, p=0.01$ ) (Table 1$)$. Among AKI patients, hospital mortality was lowest in those who had full renal recovery in the first 7 days $(22.8 \%)$ compared to patients with partial renal recovery $(59.5 \%)$ or no recovery (64\%) (Additional file 4 and Additional file 7 : Figure S3). AKI patients also had a longer length of stay in the ICU, especially if renal function did not recover.

\section{Discussion}

This retrospective analysis demonstrates that ICUacquired AKI is common and associated with a high risk of death, especially if renal function does not recover quickly. More than $30 \%$ of critically ill patients who did not have AKI on admission to the ICU developed AKI during the first 24 to $72 \mathrm{~h}$ in the ICU. Pre-existing CKD, more severe AKI, need for mechanical ventilation and diuretic use were independently associated with a higher risk of non-recovery. Cumulative fluid balance was strongly associated with both the development of AKI and non-recovery. For non-recovery of renal function, the association was $U$-shaped; both extremely negative and extremely positive cumulative fluid balance were 
Table 1 Demographic and baseline clinical parameters

\begin{tabular}{|c|c|c|c|c|}
\hline Variables* & $\begin{array}{l}\text { All patients without AKI on admission } \\
(n=2525)\end{array}$ & $\begin{array}{l}\text { New AKI in the ICU } \\
(n=840)\end{array}$ & $\begin{array}{l}\text { No new AKI in the ICU } \\
(n=1685)\end{array}$ & $\begin{array}{l}p \\
\text { value }\end{array}$ \\
\hline \multicolumn{5}{|l|}{ Demographics } \\
\hline Age & $62[48,74]$ & $64[51,74]$ & $61[43,72]$ & 0.02 \\
\hline Male sex & $1498(59.3)$ & $519(62.0)$ & $979(58.0)$ & 0.06 \\
\hline BMI & $24.2[23,28.1]$ & $25.4[23.5,30.0]$ & $24.2[22.9,29.0]$ & $\begin{array}{l}< \\
0.001\end{array}$ \\
\hline Body weight $[\mathrm{kg}]$ & $70[63,81]$ & $73[65,89]$ & $70[60,78]$ & $\begin{array}{l}< \\
0.001\end{array}$ \\
\hline \multicolumn{5}{|l|}{ Parameters on the day of ICU admission } \\
\hline Medical admission & $2012(80)$ & $677(81)$ & $1335(79)$ & 0.69 \\
\hline Elective surgical admission & $367(15)$ & $113(14)$ & $254(16)$ & \\
\hline Emergency surgical admission & $146(5)$ & $50(6)$ & $96(6)$ & \\
\hline Lowest MAP $[\mathrm{mmHg}]$ & $59[53,64]$ & $56[51,61]$ & $59[55,62]$ & 0.01 \\
\hline SOFA score & $5[3,7]$ & $5[3,8]$ & $4[3,7]$ & $\begin{array}{l}< \\
0.001\end{array}$ \\
\hline CVP $[\mathrm{mmHg}]$ & $12[8,17]$ & $13[9,18]$ & $11[7,16]$ & $<.001$ \\
\hline \multicolumn{5}{|l|}{ Comorbidities } \\
\hline Baseline serum creatinine $[\mu \mathrm{mol} / \mathrm{L})$ & $83[63,113]$ & $100[75,140]$ & $81[60,117]$ & $<.001$ \\
\hline Chronic kidney disease & $228(9.0)$ & $111(13.3)$ & $117(6.9)$ & $<.001$ \\
\hline Chronic lung disease & $715(28.3)$ & $246(29.4)$ & $469(27.8)$ & 0.40 \\
\hline Chronic liver disease & $1645(65.1)$ & $553(66.1)$ & $1092(67.1)$ & 0.51 \\
\hline Cardiovascular disease & $545(21.6)$ & $222(26.5)$ & $343(20.3)$ & $\begin{array}{l}< \\
0.001\end{array}$ \\
\hline Congestive heart failure & $200(8.0)$ & $80(9.6)$ & $120(7.1)$ & 0.04 \\
\hline Diabetes mellitus & $517(20.5)$ & $197(23.5)$ & $320(19.0)$ & 0.01 \\
\hline Cerebrovascular disease & $206(8.2)$ & $73(8.7)$ & $133(7.9)$ & 0.49 \\
\hline Cancer & $730(28.9)$ & $218(26.0)$ & $512(30.3)$ & 0.03 \\
\hline \multicolumn{5}{|c|}{ Primary diagnostic code for ICU admission } \\
\hline Respiratory & 899 (35.6) & $298(35.6)$ & $601(35.6)$ & 1.00 \\
\hline Neurologic & $179(7.1)$ & $61(7.3)$ & $118(7.0)$ & 0.81 \\
\hline Post-surgery & $429(17.0)$ & $132(15.8)$ & $297(17.6)$ & 0.26 \\
\hline Cardiovascular & $391(15.4)$ & $145(17.3)$ & $246(14.6)$ & 0.08 \\
\hline Gastrointestinal & $183(7.2)$ & $59(7.0)$ & $124(7.3)$ & 0.81 \\
\hline Urinary & $57(2.3)$ & $14(1.7)$ & $43(2.5)$ & 0.20 \\
\hline Sepsis & $210(8.3)$ & $84(10.0)$ & $126(7.5)$ & 0.03 \\
\hline Other & $339(13.4)$ & $109(13.0)$ & $230(13.6)$ & 0.71 \\
\hline \multicolumn{5}{|c|}{ Organ support from ICU admission to the day of AKI or day 3} \\
\hline Mechanical ventilation & $1444(57.2)$ & $512(61.2)$ & $932(55.2)$ & 0.04 \\
\hline ECMO & $130(5.1)$ & $61(7.3)$ & $69(4.1)$ & 0.01 \\
\hline IABP & $62(2.5)$ & $31(3.7)$ & $31(1.8)$ & 0.01 \\
\hline Surgery & $101(4.0)$ & $35(4.2)$ & $66(3.9)$ & 0.75 \\
\hline Epinephrine & $27(1.1)$ & $13(1.6)$ & $14(0.8)$ & 0.10 \\
\hline Norepinephrine & 1050 (41.6) & $427(50.8)$ & $627(37.1)$ & $\begin{array}{l}< \\
0.001\end{array}$ \\
\hline
\end{tabular}


Table 1 Demographic and baseline clinical parameters (Continued)

\begin{tabular}{|c|c|c|c|c|}
\hline Variables* & $\begin{array}{l}\text { All patients without AKI on admission } \\
(n=2525)\end{array}$ & $\begin{array}{l}\text { New AKI in the ICU } \\
(n=840)\end{array}$ & $\begin{array}{l}\text { No new AKI in the ICU } \\
(n=1685)\end{array}$ & $\begin{array}{l}p \\
\text { value }\end{array}$ \\
\hline Vasopressin & $9(0.4)$ & $5(0.6)$ & $4(0.2)$ & 0.17 \\
\hline \multicolumn{5}{|c|}{ Time (in the first 7 days) spent on inotropic and vasoactive medications } \\
\hline $\begin{array}{l}\text { Number of days on at least } 1 \text { inotrope or } \\
\text { vasopressor }\end{array}$ & $0[0,0]$ & $1[0,3]$ & $0[0,2]$ & $\begin{array}{l}< \\
0.001\end{array}$ \\
\hline 0 days & $1405(56)$ & $382(45)$ & $1023(61)$ & \\
\hline $1-3$ days & $725(29)$ & $252(30)$ & $473(28)$ & \\
\hline 4 or more days & $395(16)$ & $206(25)$ & $189(11)$ & \\
\hline $\begin{array}{l}\text { Number of days on }>1 \text { inotrope or } \\
\text { vasopressor }\end{array}$ & $0[0,0]$ & $0[0,0]$ & $0[0,0]$ & $\begin{array}{l}< \\
0.001\end{array}$ \\
\hline 0 days & $2324(92)$ & $737(88)$ & $1587(94)$ & \\
\hline $1-3$ days & $178(7)$ & $87(10)$ & $91(5)$ & \\
\hline 4 or more days & $23(1)$ & $16(2)$ & $7(0)$ & \\
\hline Number of days on at least 1 vasopressor & $0[0,0]$ & $1[0,3]$ & $0[0,2]$ & $\begin{array}{l}< \\
0.001\end{array}$ \\
\hline 0 days & $1428(57)$ & $386(46)$ & $1042(62)$ & \\
\hline $1-3$ days & $718(28)$ & $251(30)$ & $467(28)$ & \\
\hline 4 or more days & $379(15)$ & $203(24)$ & $176(11)$ & \\
\hline Number of days on $>1$ vasopressor & $0[0,0]$ & $0[0,0]$ & $0[0,0]$ & 0.001 \\
\hline 0 days & $2502(99)$ & $825(98)$ & $1677(99)$ & \\
\hline $1-3$ days & $23(1)$ & $15(2)$ & $8(1)$ & \\
\hline 4 or more days & 0 & 0 & 0 & \\
\hline \multicolumn{5}{|l|}{ Potentially nephrotoxic exposures } \\
\hline Vancomycin & $240(9.5)$ & $82(9.8)$ & $158(9.4)$ & 0.72 \\
\hline Diuretic & $1185(46.9)$ & $381(45.5)$ & $804(47.6)$ & 0.33 \\
\hline Aminoglycosides & $899(35.6)$ & $291(34.8)$ & $608(36.0)$ & 0.57 \\
\hline ACE-I/ARB & $180(7.1)$ & $67(8.0)$ & $113(6.7)$ & 0.25 \\
\hline Contrast & $252(10.0)$ & $88(10.5)$ & $164(9.7)$ & 0.53 \\
\hline Chemotherapy & $42(1.7)$ & $11(1.3)$ & $31(1.8)$ & 0.88 \\
\hline Antiretroviral drugs & $40(1.6)$ & $11(1.3)$ & $30(1.8)$ & 0.41 \\
\hline NSAID & $51(2.0)$ & $8(1.0)$ & $43(2.5)$ & 0.01 \\
\hline \multicolumn{5}{|l|}{ Fluid balance on the day of AKI/day 3} \\
\hline Cumulative FB in ml & $1389(3455)$ & $2148.4(3667.5)$ & $1002(3276)$ & $\begin{array}{l}< \\
0.001\end{array}$ \\
\hline$\%$ of fluid balance in $\%$ BW & $1.66[-0.7,4.3]$ & $3.98[1.2,6.9]$ & $2.29[-0.07,5.14]$ & $\begin{array}{l}< \\
0.001\end{array}$ \\
\hline \multicolumn{5}{|l|}{ Outcomes } \\
\hline ICU stay (days) & & $13[9,21]$ & $9[5,17]$ & $<0.01$ \\
\hline Hospital stay (days) & & $31[19,56]$ & $22[12,43]$ & 0.94 \\
\hline ICU mortality (\%) & & $175(20.9)$ & $94(5.6)$ & $<0.01$ \\
\hline Hospital mortality (\%) & & $273(35.4)$ & $243(15.4)$ & $<0.01$ \\
\hline
\end{tabular}

$A C E-l$ angiotensin converting enzyme inhibitor, $A R B$ angiotensin receptor blocker, $A K l$ acute kidney injury, $B M I$ body mass index, $B W$ body weight, $C V P$ central venous pressure, ECMO extracorporeal membrane oxygenation, $F B$ fluid balance, IABP intra-aortic balloon pump, ICU intensive care unit, MAP mean arterial pressure, NSAID non-steroidal anti-inflammatory drug, SD standard deviation, SOFA Sequential Organ Failure Assessment

*Results displayed as $n(\%)$, mean (SD) or median [interquartile range] 
Table 2 Multivariate analysis of risk factors for the development of new acute kidney injury

\begin{tabular}{|c|c|c|c|c|}
\hline \multirow[t]{2}{*}{ Variables } & \multirow[t]{2}{*}{ OR } & \multicolumn{2}{|c|}{$95 \% \mathrm{Cl}$ for OR } & \multirow{2}{*}{$\begin{array}{l}p \\
\text { value }\end{array}$} \\
\hline & & Lower & Upper & \\
\hline Age & 1.00 & 1.00 & 1.01 & 0.24 \\
\hline BMI & 1.06 & 1.04 & 1.07 & $<0.001$ \\
\hline SOFA score on admission to the ICU & 1.04 & 1.00 & 1.08 & 0.05 \\
\hline Lowest MAP & 1.00 & 0.99 & 1.01 & 0.84 \\
\hline Sepsis & 1.11 & 0.80 & 1.53 & 0.53 \\
\hline Chronic kidney disease & 1.81 & 1.34 & 2.45 & $<0.001$ \\
\hline Atherosclerotic cardiovascular disease & 1.13 & 0.90 & 1.40 & 0.29 \\
\hline Congestive heart failure & 1.12 & 0.81 & 1.56 & 0.50 \\
\hline Diabetes mellitus & 1.06 & 0.85 & 1.33 & 0.60 \\
\hline Cancer & 0.84 & 0.69 & 1.03 & 0.10 \\
\hline Mechanical ventilation & 0.94 & 0.77 & 1.17 & 0.60 \\
\hline Norepinephrine use & 1.20 & 0.94 & 1.54 & 0.15 \\
\hline NSAID use & 0.48 & 0.22 & 1.05 & 0.07 \\
\hline Cumulative fluid balance on the day of AKI or day 3 in the ICU & 1.11 & 1.08 & 1.14 & $<0.001$ \\
\hline
\end{tabular}

\section{Model diagnostics:}

There was no evidence of lack of fit (Hosmer Lemeshow $p=0.209$ ) that the model was incorrectly specified (link test $p=0.300)$ or of multicollinearity among the included covariates (all variance inflation factors (VIF) < 1.5). There was no evidence of a non-linear relationship between cumulative fluid balance and AKI $B M I$ body mass index, Cl confidence interval, MAP mean arterial pressure, NSAID non-steroidal anti-inflammatory drug, SOFA Sequential Organ Failure Assessment, OR odd ratio

harmful to the kidney function. Finally, the chances of being discharged alive from the hospital were significantly better in patients who either did not develop AKI or recovered renal function within 1 week compared to those who still had AKI on day 7.

Although it is not always possible to modify risk factors for AKI, many episodes of AKI are considered to be preventable. It is therefore essential to identify those risk factors that can either be avoided or altered. In our analysis, cumulative fluid balance emerged as a strong risk factor with a U-shape association. Both inadequate fluid administration and fluid accumulation were independently associated with an increased risk of non-recovery. Cumulative fluid balance by the day of AKI (or day 3 in

Table 3 Multivariate analysis for risk of non-recovery after acute kidney injury

\begin{tabular}{|c|c|c|c|c|c|c|c|c|}
\hline \multirow[t]{3}{*}{ Variables } & \multicolumn{4}{|c|}{ MVR (variables pre-AKI) ${ }^{1}$} & \multicolumn{4}{|c|}{ MVR (variables post-onset of AKI) ${ }^{2}$} \\
\hline & \multirow[t]{2}{*}{ OR } & \multicolumn{2}{|c|}{$95 \% \mathrm{Cl}$ for OR } & \multirow{2}{*}{$\begin{array}{l}p \\
\text { value }\end{array}$} & \multirow[t]{2}{*}{ OR } & \multicolumn{2}{|c|}{$95 \% \mathrm{Cl}$ for OR } & \multirow[t]{2}{*}{$p$ value } \\
\hline & & Lower & Upper & & & Lower & Upper & \\
\hline AKI stage & & & & & 1 & & & $<0.001$ \\
\hline AKI stage 2 & & & & & 0.63 & 0.31 & 1.28 & 0.21 \\
\hline AKI stage 3 & & & & & 2.24 & 1.16 & 4.31 & 0.02 \\
\hline SOFA score on admission to the ICU & 1.05 & 0.96 & 1.15 & 0.26 & & & & \\
\hline SOFA score on the day of AKI/day 3 & - & - & - & - & 1.10 & 0.99 & 1.22 & 0.09 \\
\hline Lowest MAP on the day of AKI & - & - & - & - & 0.99 & 0.96 & 1.02 & 0.36 \\
\hline Chronic kidney disease & 2.01 & 1.12 & 3.58 & 0.02 & 2.82 & 1.37 & 5.78 & 0.01 \\
\hline Reason for admission: respiratory & 1.52 & 0.94 & 2.45 & 0.09 & & & & \\
\hline Mechanical ventilation & 2.29 & 1.24 & 4.26 & 0.01 & 4.34 & 2.05 & 9.15 & $<0.001$ \\
\hline Norepinephrine use & 1.21 & 0.64 & 2.30 & 0.56 & 0.56 & 0.25 & 1.26 & 0.16 \\
\hline Vancomycin use & 1.10 & 0.48 & 2.48 & 0.83 & 1.40 & 0.65 & 3.01 & 0.39 \\
\hline Diuretic use & 1.89 & 1.20 & 2.97 & 0.01 & & & & \\
\hline Aminoglycoside use & 1.13 & 0.69 & 1.83 & 0.63 & & & & \\
\hline
\end{tabular}

Model diagnostics:

There was no evidence of lack of fit (Hosmer Lemeshow $p=0.217$ and 0.248 ) that the model was incorrectly specified (link test $p=0.082$ and 0.345 ) or of multicollinearity among the included covariates (all variance inflation factors $<3$ ) for model MVR 1 or MVR 2

$A K I$ acute kidney injury, Cl confidence interval, MAP mean arterial pressure, ICU intensive care unit, MVR multivariate analysis, NSAID non-steroidal antiinflammatory drug, SOFA Sequential Organ Failure Assessment, OR odd ratio

${ }^{1}$ Also adjusted for the non-linear association between fluid balance and non-recovery $(p<0.001)$

${ }^{2}$ Also adjusted for the non-linear association between net fluid balance and non-recovery $(p=0.016)$ 

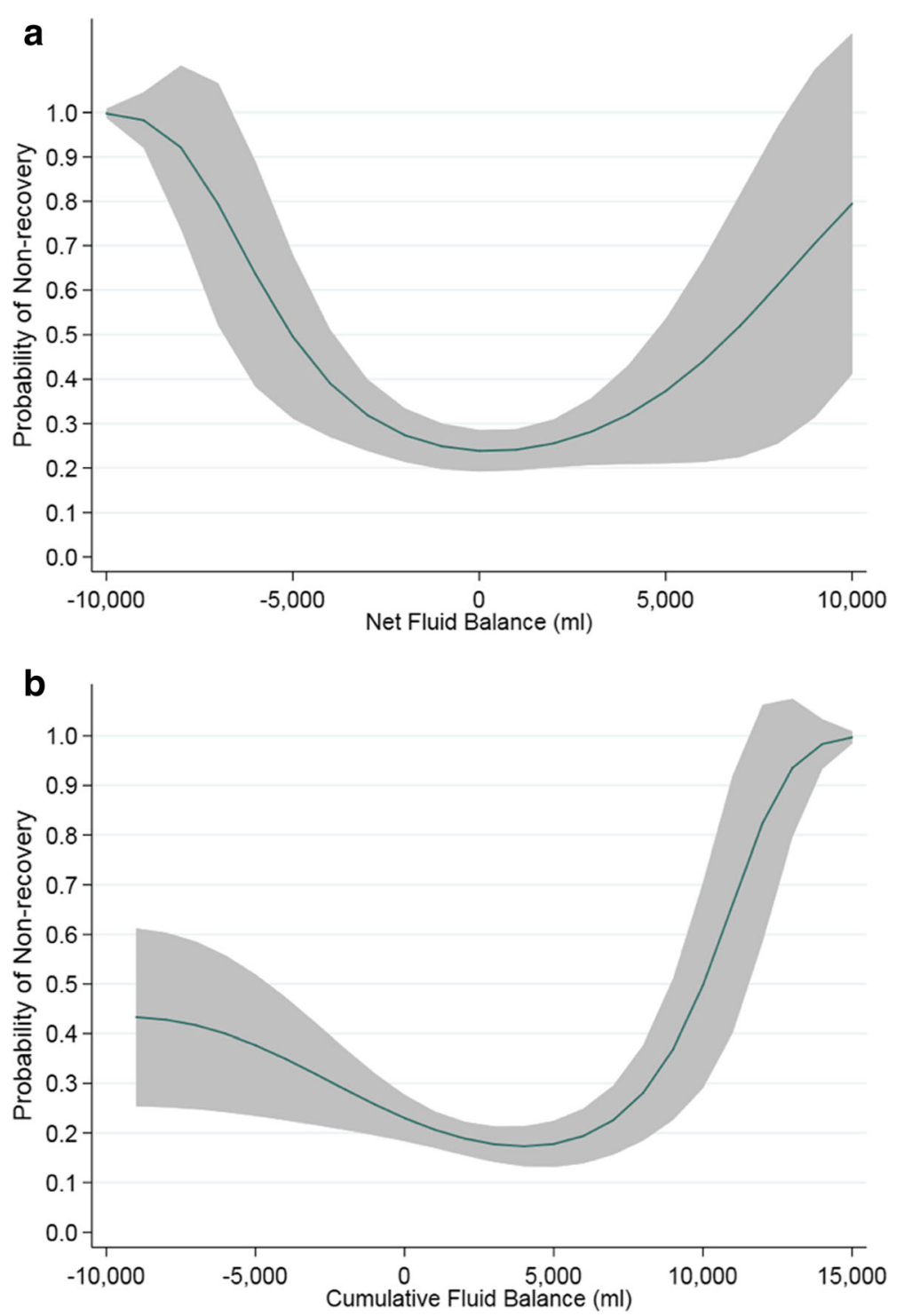

Fig. 2 Association between fluid balance and non-recovery of renal function. a Association between net fluid balance on the first day of AKI and probability of renal non-recovery. $\mathbf{b}$ Association between cumulative fluid balance at $48 \mathrm{~h}$ after the onset of AKI and probability of renal non-recovery

the ICU) was also strongly associated with the development of new AKI. Raimundo et al. previously showed that increased fluid administration in early AKI was an independent risk factor for progression to AKI stage III [11]. Data from the Sepsis Occurrence in Acutely Ill Patients demonstrated a $54 \%$ adjusted risk of death at 60 days in patients with AKI and a mean positive fluid balance [12]. Although the exact reasons for the deleterious impact of fluid overload on renal function are not known, obstruction of capillary blood flow and lymphatic drainage, renal congestion and impaired tissue oxygenation are likely to play an important role [13, 14]. These effects are particularly pronounced in encapsulated organs such as the liver and kidneys that lack the capacity to accommodate extra volume without an increase in the interstitial pressure. Importantly, there is no evidence that fluid accumulation is beneficial or necessary in AKI or during acute illness. Van Biesen et al. previously showed that additional fluid loading not only failed to improve renal function but was also associated with worsening respiratory function [15].

Fluid management is a key component of prevention and management of AKI $[9,16]$. Although we showed that both negative and positive fluid balance were associated with worse outcomes, it is important to note that our study and others in the literature only demonstrate associations but do not prove a causal relationship [11, 12, 17]. It is certainly possible that there was more 
haemodynamic instability and hypotension among those who received larger volumes of fluid and accumulated more fluid and that haemodynamic instability per se may have contributed to the risk of AKI. The role of active fluid restriction in AKI with focus on the prevention of fluid overload has not been studied prospectively. However, data from the CLASSIC feasibility study showed that a protocol of restricting fluid administration after initial resuscitation in patients with septic shock was safe and associated with less worsening of AKI compared to standard care [18]. Until more data are available, our results, together with reports in the literature suggest that regular assessment of both daily fluid balance and cumulative fluid balance are crucial. Whilst fluid administration is an important component of the initial resuscitation phase and serves to prevent AKI in patients with intravascular hypovolaemia, there comes a point when fluids have a deleterious effect on renal function. Unfortunately, the exact timing and how best to identify the time point remain unclear.

Another interesting result of our analysis was the emergence of obesity as an independent risk factor for the development of AKI. Previous studies showed a higher risk of AKI in trauma and acute lung injury patients with higher BMI [19-21]. Others pointed out that the association between BMI and risk of AKI appeared to be a "U"-shaped curve with both under- and overweight patients being particularly at risk [22]. Billings et al. speculated that the association between obesity and AKI after cardiac surgery might be mediated by oxidative stress [23]. It is also possible that inappropriate dosing of nephrotoxic drugs in patients with a higher BMI plays a role. However, it is also important to acknowledge that the utilization of weight-based urine volume criteria may overestimate the risk of AKI in patients with a higher BMI resulting in an overestimation of AKI [24].

To the best of our knowledge, this study is the first to describe the non-linear relationship of cumulative FB and non-recovery of renal function. Both extremely negative and extremely positive FB were independently associated with non-recovery whilst increased FB was associated with the development of new AKI. These results should serve to underpin a future intervention trial exploring the role of fluid restriction in AKI.

Our analysis has some limitations which we would like to acknowledge. Due to the retrospective nature of the analysis, we were limited to the data that had been collected for clinical reasons. For instance, CVP measurements were not performed in all patients who developed AKI. As a result, we were not able to explore the relationship between CVP and AKI. Secondly, we defined ICU-acquired AKI as AKI that had developed at least 24 $h$ after ICU admission. The decision was based on similar approaches in the literature [10]. However, we acknowledge that serum creatinine and urine output are relatively late indicators of $\mathrm{AKI}$ and that in a proportion of patients categorized with "AKI after ICU admission", the onset of AKI may have been before ICU admission. In the future, the implementation of new AKI biomarkers may help to identify the onset of AKI more accurately and the AKI consensus definition and staging classification may be revised accordingly. Third, we excluded patients who developed AKI during the 72-h to 7-day period and acknowledge that they potentially had ICU-acquired AKI. The reason for excluding them was that we intended to analyse chances of renal recovery at day 7 and, therefore, we needed to allow patients with AKI a minimum time for kidney function to recover. Fourth, we evaluated renal recovery on day 7 . The main reason was that we did not have complete data beyond this time period. We acknowledge that a proportion of patients may have either developed AKI later or recovered AKI after day 7. Fifth, in our models assessing factors associated with the development of new AKI, we included cumulative FB on the day of AKI (or day 3 in the ICU for those without AKI) and it is possible that the increased cumulative FB in those with AKI may be a consequence of AKI itself rather than a risk factor for AKI. Sixth, we used creatinine results without correcting for fluid balance. We acknowledge that AKI definition and classification could change with adjustment for fluid balance [25]. However, the current AKI consensus definition is based on creatinine values without correction. Finally, our data stem from a large multi-disciplinary ICU in a single centre. Therefore, our conclusions may not be generalizable to other patient populations.

\section{Conclusions}

ICU-acquired AKI is associated with a high risk of mortality, especially if renal function does not recover by day 7. Cumulative fluid balance is a strong independent risk factor for the development and non-recovery of AKI with a U-shape relationship.

\section{Supplementary information}

Supplementary information accompanies this paper at https://doi.org/10. 1186/s13054-019-2673-5.

Additional file 1: Association between parameters before $A K I$ and subsequent AKI recovery.

Additional file 2: Association between parameters during 24-48 hours period after $\mathrm{AKI}$ and $\mathrm{AKI}$ recovery.

Additional file 3: Comparison between patients with extremes of cumulative fluid balance on day of AKI or day 3 in ICU.

Additional file 4: Table S1. Outcomes in AKI with and without renal recovery

Additional file 5: Figure S1. Association between cumulative fluid balance on day of AKI and probability of non-recovery of renal function. 
Additional file 6: Figure S2. Association between net fluid balance after 24 hours in ICU and probability of non-recovery of renal function.

Additional file 7: Figure S3. Survival curves of AKI and non-AKI patients.

\section{Abbreviations}

ACE-I: Angiotensin converting enzyme inhibitor; ARB: Angiotensin receptor blocker; AKI: Acute kidney injury; BMI: Body mass index; BW: Body weight; Cl: Confidence interval; CKD: Chronic kidney disease; CRRT: Continuous renal replacement therapy; CVP: Central venous pressure; ECMO: Extracorporeal membrane oxygenation; ESRD: End-stage renal disease; FB: Fluid balance; IABP: Intra-aortic balloon pump; ICU: Intensive care unit; IQR: Interquartile ratio; KDIGO: Kidney Disease Improving Global Outcome; MAP: Mean arterial pressure; NSAID: Non-steroidal anti-inflammatory drug; REC: Research Ethics Committee; RRT: Renal replacement therapy; SD: Standard deviation;

SOFA: Sequential Organ Failure Assessment

\section{Authors' contributions}

MO developed the research protocol and oversaw the project, including data collection, data analysis and drafting of the manuscript. JZ contributed to the protocol, collected the data, performed the first analysis and wrote the first draft of the paper. SC is a senior statistician in a clinical trials unit She oversaw the statistical analyses and helped interpreting the data. AD contributed to the data collection and interpretation of the results. NS contributed to the data collection and interpretation of the results. ZYP contributed to the interpretation of the results. All authors helped revising the paper and read and approved the final manuscript.

\section{Funding}

None.

\section{Availability of data and materials}

The datasets used and analysed during the current study are available from the corresponding author on reasonable request

\section{Ethics approval and consent to participate}

The study had institutional approval. Need for individual informed consent was waived as this was a retrospective analysis of data collected prospectively for routine care, and there was no breach of privacy or anonymity (UK National Research Ethics Service).

\section{Consent for publication}

Not applicable.

\section{Competing interests}

The authors declare that they have no competing interests.

\section{Author details}

'Department of Critical Care, King's College London, Guy's and St Thomas' NHS Foundation Trust, London SE1 7EH, UK. ²Department of Critical Care Medicine, Zhongnan Hospital of Wuhan University, Wuhan, China. ${ }^{3}$ Medical Research Council Clinical Trials Unit, University College London, London, UK ${ }^{4}$ Department of Critical Care, Guy's and St Thomas' NHS Foundation Trust, London, UK. ${ }^{5}$ Sechenov Biomedical Science and Technology Park, Sechenov First Moscow State Medical University, Moscow, Russian Federation.

Received: 24 September 2019 Accepted: 13 November 2019 Published online: 03 December 2019

\section{References}

1. Hoste EA, Bagshaw SM, Bellomo R, et al. Epidemiology of acute kidney injury in critically ill patients: the multinational AKI-EPI study. Intensive Care Med. 2015:41(8):1411-123.

2. Ostermann M, Joannidis M. Acute kidney injury in 2016: diagnosis and diagnostic workup. Crit Care. 2016;20(1):299.

3. Ostermann M, Cerda J. The burden of AKI and related financial issues. Contrib Nephrol. 2018;193:100-12.

4. Chawla LS, Davison DL, Brasha-Mitchell E, et al. Association between AKI and long-term renal and cardiovascular outcomes in United States veterans. Clin J Am Soc Nephrol. 2014;9(3):448-56.
5. Wald R, Quinn RR, Adhikari NK, et al. Risk of chronic dialysis and death following acute kidney injury. Am J Med. 2012;125:585-93.

6. Guerin C, Girard R, Selli JM, et al. Initial versus delayed acute renal failure in the intensive care unit. A multicenter prospective epidemiological study. Rhone-Alpes Area Study Group on Acute Renal Failure. Am J Respir Crit Care Med. 2000;161(3 Pt 1):872-9.

7. Meersch M, Schmidt C, Hoffmeier A, et al. Prevention of cardiac surgeryassociated AKI by implementing the KDIGO guidelines in high risk patients identified by biomarkers: the PrevAKI randomized controlled trial. Intensive Care Med. 2017:43(11):1551-61.

8. Selby NM, Casula A, Lamming $L$, et al. An organizational-level program of intervention for AKI: a pragmatic stepped wedge cluster randomized trial. J Am Soc Nephrol. 2019:30(3):505-15.

9. Kidney Disease: Improving Global Outcomes (KDIGO) Acute Kidney Injury Work Group. KDIGO clinical practice guideline for acute kidney injury. Kidney Int. 2012;2:1-138.

10. Abd ElHafeez S, Tripepi G, Quinn R, Naga Y, Abdelmonem S, AbdelHady M, Liu P, James M, Zoccali C, Ravani P. Risk, predictors, and outcomes of acute kidney injury in patients admitted to intensive care units in Egypt. Sci Rep. 2017;7(1):17163.

11. Raimundo M, Crichton S, Martin JR, et al. Increased fluid administration after early acute kidney injury is associated with less renal recovery. Shock. 2015; 44(5):431-7.

12. Payen D, de Pont AC, Sakr Y, et al. A positive fluid balance is associated with a worse outcome in patients with acute renal failure. Crit Care. 2008; 12(3):R74.

13. Prowle JR, Bellomo R. Fluid administration and the kidney. Curr Opin Crit Care. 2013:19(4):308-14.

14. Firth JD, Raine AE, Ledingham JG. Raised venous pressure: a direct cause of renal sodium retention in oedema? Lancet. 1988;1 (8593):1033-5.

15. Van Biesen W, Yegenaga I, Vanholder $\mathrm{R}$, et al. Relationship between fluid status and its management on acute renal failure (ARF) in intensive care unit (ICU) patients with sepsis: a prospective analysis. J Nephrol. 2005;18(1): 54-60.

16. Ostermann M, Liu K, Kashani K. Fluid management in acute kidney injury. Chest. 2019;156(3):594-603.

17. Ostermann M, Oudemans van-Straaten HM, Forni LG. Fluid overload and acute kidney injury: cause or consequence? Crit Care. 2015;19(1):443.

18. Hjortrup $\mathrm{PB}$, Haase $\mathrm{N}$, Bundgaard $\mathrm{H}$, et al. Restricting volumes of resuscitation fluid in adults with septic shock after initial management: the CLASSIC randomised, parallel-group, multicentre feasibility trial. Intensive Care Med. 2016:42(11):1695-705.

19. Shashaty MG, Meyer NJ, Localio AR, et al. African American race, obesity, and blood product transfusion are risk factors for acute kidney injury in critically ill trauma patients. J Crit Care. 2012;27(5):496-504.

20. Danziger J, Chen KP, Lee J, et al. Obesity, acute kidney injury, and mortality in critical illness. Crit Care Med. 2016;44(2):328-34.

21. Soto GJ, Frank AJ, Christiani DC, et al. Body mass index and acute kidney injury in the acute respiratory distress syndrome. Crit Care Med. 2012;40(9): 2601-8.

22. Zou Z, Zhuang Y, Liu L, et al. Role of body mass index in acute kidney injury patients after cardiac surgery. Cardiorenal Med. 2017:8(1):9-17.

23. Billings FT 4th, Pretorius M, Schildcrout JS, et al. Obesity and oxidative stress predict AKI after cardiac surgery. J Am Soc Nephrol. 2012;23(7):1221-8.

24. Fliser D, Laville M, Covic A, et al. A European Renal Best Practice (ERBP) position statement on the Kidney Disease Improving Global Outcomes (KDIGO) clinical practice guidelines on acute kidney injury: part 1: definitions, conservative management and contrast-induced nephropathy. Nephrol Dial Transplant. 2012;27(12):4263-72.

25. Liu KD, Thompson BT, Ancukiewicz M, et al. Acute kidney injury in patients with acute lung injury: impact of fluid accumulation on classification of acute kidney injury and associated outcomes. Crit Care Med. 2011;39(12): 2665-71.

\section{Publisher's Note}

Springer Nature remains neutral with regard to jurisdictional claims in published maps and institutional affiliations. 